\title{
Experimental Investigations of Critical Hydraulic Gradients for a Soil prone to Suffusion
}

\author{
M.R. Salehi Sadaghiani \& K.J. Witt \\ Department of Geotechnical Engineering \\ Bauhaus-Universität Weimar, Germany
}

B. Odenwald
Federal Waterways Engineering and Research Institute (BAW)
Department of Geotechnical Engineering, Karlsruhe, Germany

\begin{abstract}
The presence of soils, which are at the limit state of internal stability, is a potential risk to earthworks under seepage flow. Therefore, it is necessary to identify unstable soils and to estimate hydraulic gradients at which the suffusion can be initiated respectively progressed. An experimental study has been carried out to quantify critical hydraulic gradients for a widely graded soil. For the tested soil, in downwards vertical percolation experiments, the global critical hydraulic gradients lie in the different ranges between $i_{\text {crit }}=0.1$ to 5.5 with dependency on the particle arrangement. The critical hydraulic gradient was investigated using various types of sample preparation technique. Moreover, suffusion tests using several types of samples with the same particle size distribution have been carried out. It states that for such a widely graded soil, the main problem is the particle arrangement. In other words, the suffusion might be not problematic if there is no segregation. Sometimes a specific amount of segregation also stabilizes the sample against suffusion. The common way of sample preparation delivers comparable results to the results of other researchers.
\end{abstract}

\section{INTRODUCTION}

\subsection{Overview}

The effect of particle arrangement in microstructures is widely understood to be a major feature in controlling the behavior of all materials. In soil, this is the most important issue because of its three-phase structure and its local variations. Often non-homogeneity dispersed in a segregation of particles leads to a deformable matrix with reduced mechanical and hydraulic properties. Homogeneous distributions of the soil particles are well documented as improving the soils mechanical and hydraulic characteristics when compared to segregated soils (Saucke et al. 1999). For instance, the microstructure of the soil body has a decisive influence on the internal stability and the local hydraulic gradients of widely graded soils. In the recent years, different soil placement techniques and specifications for preparation of soil materials have been employed to improve homogeneity.

However, there are still reports of dam and embankment failures (Bonelli 2012). To ensure a more uniform average property due to uncertainties, the thick- ness of soil layers, even of filter layers, has been increased by guidelines. Generally, the homogeneous distribution of the particles at the microstructure level is unpredictable, despite all of the current considerations. The reality of the particle arrangement is much more complicated, and in-situ particle distributions are highly dependent on placement parameters during construction (i.e., the moisture content of the soil, drop height, geometry, particle coarseness, the relation between particle diameters and vibrations). There is a general assumption in geotechnical communities that the particle arrangement of poorly graded soils, like filter materials, is homogeneous. Even in such a case, after (Kenney and Westland 1993), the poorly graded soils are also vulnerable to segregation.

Different internal erosion failure modes may occur within a dam, river embankment or in their subsoil/foundation by various mechanisms (Richards and Reddy 2007) The term "internal erosion" is generally used to specify any process that causes erosion within a soil body which cannot be seen. Several different internal erosion failure modes have been defined in the literature, as follows: 
1. Backward erosion: the process of forming a pipe by progressive erosion starting at an exit point and operating backward towards the upstream water or the surface of the earthwork. In the literature, there is also a process named Suffosion which is defined as a process similar to backward erosion that occurs in a widely gap graded soil without the skeleton, i.e. the soil has a dominant fine matrix (see Jentsch et al. 2014). Authors consider this process as the backward erosion process.

2. Hydraulic heave: the process of lifting a soil mass or soil particles as a result of an imbalance between seepage uplift forces and the buoyant weight of the soil particles.

3. Concentrated leak erosion (scour): the process whereby erosion initiated along the walls of a crack, geological or constructional contact, or voids generated by settlement or construction defects.

4. Tunneling: usually the process of zone desaturation in natural slopes or a process that occurs in earthworks or foundations composed of dispersive soils (Sherard et al. 1977).

5. Contact erosion: the process of particle transport between two soil layers, i.e. a cohesive and a non-cohesive soil (base-filter-combination) in the contact area, if there is a crack in a cohesive soil layer, which cannot be repaired by itself. The risk of contact erosion can be checked, for instance, by the method of Sherard \& Dunnigan (1989).

6. Suffusion: the process in widely and gap-graded soils with a dominant coarse matrix (soils with a higher percentage of coarse grains which build the soil skeleton and fines embedded within the pore spaces of the soil skeleton), whereby finer particles suffuse through the pores between coarser skeletal grains (see also Jentsch et al. 2014).

While each of these definitions describes a specific seepage related failure mode, they might also take place in some combination or occur at different locations within the earthwork or its foundation. In risk assessments of potential damage to earthwork, the consideration of erosion processes caused by seepage through or under an earthwork is of great importance. This is especially valid if the subsoil or the dam body consists of soil prone to suffusion. These are widely graded soils with a higher coefficient of uniformity $\left(c_{u}>15\right)$. In such a soil, particle transport starts at much lower hydraulic gradients than in soils with a smaller coefficient of uniformity $\left(c_{u}<15\right)$. It is because of the natural segregation which occurs during the soil placement and compaction. A much higher hydraulic gradient is sometimes needed for mobilization of the fine particle if there is segregation. The result of particle transport is the washout of the potentially mobile particles (loose particles). It has not been clear how important the effect of soil arrangement on the critical gradients or critical filter velocity is. Therefore, a special testing device was designed to investigate the particle transport phenomenon dependent on the factors mentioned above.

\subsection{State of the art}

Generally, to assess whether suffusion is theoretically likely or not, the particle diameters which determine the geometry of the pore channels, have to be investigated. According to the bi-modal theory for widely graded soils, suffusion is only possible if the particles of the secondary fabric (particles which fill the pores of the primary fabric or skeleton) can pass through the pores of the skeleton (Ziems.J. 1969; Kézdi 1979; Kenney and Lau 1985; Kenney and Lau 1986; Burenkova 1993; Wan and Fell 2008; Witt (2013)) Since the pore channel geometry cannot be exactly measured, the commonly used assessment methods are based on the soil PSD either analytically or numerically. There are also some methods for the calculation of the constriction size distribution (CSD) of the soils. Nevertheless, both approaches are designed using a few assumptions (Reboul et al. 2010; To et al. 2012).

In the last decade, several studies have been devoted to developing geometrical or hydraulical suffusion criteria, and there has been much less researching attention to the soil homogeneity. As a matter of fact, the conditions in the laboratory and the field are not comparable. Using pressure sensors or triaxial test apparatus, one has more access to local hydraulic gradients and the distribution of the critical hydraulic gradient $\left(i_{c r i t}\right)$. Perzlmaier (2007) observed that the use of the local hydraulic gradient improves the precision of suffusion initiation. Moffat et al. (2011) defined the occurrence of internal instability by the decrease with time in local hydraulic gradient. However, Marot et al. (2011) showed that the variations of the local hydraulic gradient can be very different by several orders of magnitude according to the local heterogeneity of the specimen. The high variation of the local hydraulic gradients addresses the effect of heterogeneity and spatial variability. Marot et al. (2011) showed that the $i_{c r i t}$ must be determined based on variations of both seepage velocity and hydraulic gradient (or pressure gradient). Different researchers observed that the value of the $i_{\text {crit }}$ required to initiate suffusion, decreases with the length of the specimen tested, which implies that the Representative Elementary Volume (REV) of the sample was not fully satisfied. Thus, it is necessary to study the suffusion process within a definite REV-size under a controlled effective stress regarding its local and global variabil- 


\subsection{Gometrical suffusion citerion}

Suffusion is the transport and washout of the fill particles of a soil through the soil skeleton. It can be modeled as a contact erosion process between fill and skeleton fractions of the soil. There are several criteria for assessment of the geometrical suffusion condition for various types of soil (Kézdi 1979; Kenney and Lau 1986; Chapuis 1992). Witt (2013) have developed a geometrical suffusion criterion based on many different investigations for a definite range of widely graded soils used in the upper Rhein river embankments. Witt proposed splitting up the PSD and assessing the stability by Terzaghi's well-known filter rule.

Kenney and Lau (1985) proposed transforming the PSD curve into a $F-H$ diagram. Where $F$ is the mass percentage of particles with diameters less than a particular diameter $d$ and $H$ is the mass percentage of particles with diameters between $d$ and $4 d$. If $H / F \geq 1.0$ the soil is internally stable (no suffusion). It implies that all of the fractions from the soil load bearing structure. An example of the $F-H$ diagram which is calculated for the investigated soil is shown in Figure 2. The soil PSD is given in Figure 1

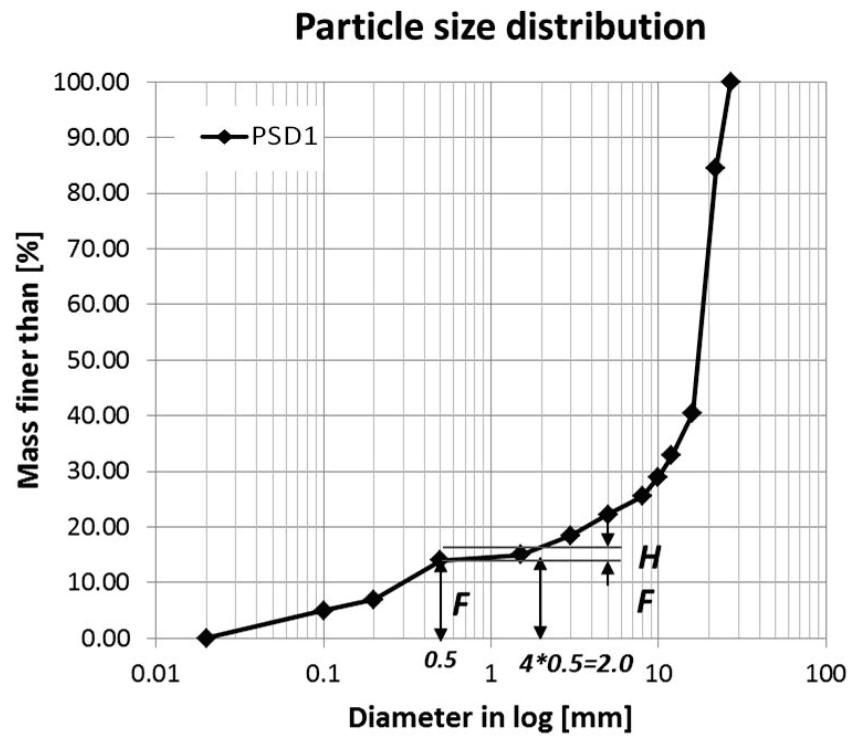

Figure 1: Investigated PSD with marked minimum amount of $\mathrm{H} / \mathrm{F}$

Chapuis (1992) simplified Kezdi's and Kenny and Lau's criteria which consider the slope of the gradation curve. He stated that the soil is internally unstable if the slope of the PSD is flatter than $15 \%$ per four times change in particle diameter, i.e $H \leq 0.15$ for all F values, Witt (2013) developed a suffusion criterion in which a separation point must be considered. The separation point divides the PSD into coarse and fine fractions. If the soil is prone to suffusion, the coarse fractions form the load-bearing skeleton and the fine fractions cannot fill totally the pore spaces of

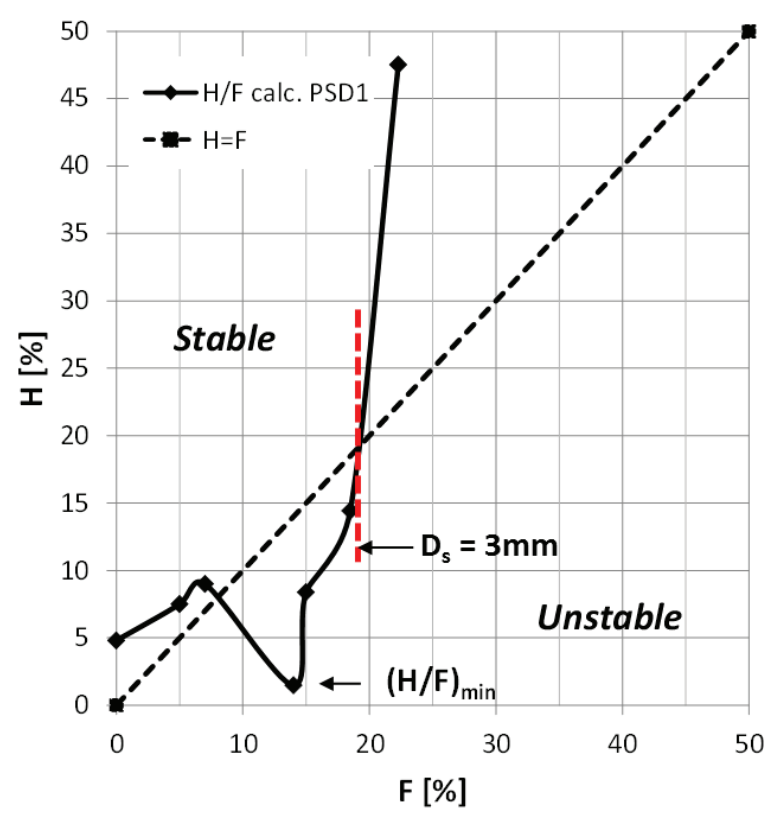

Figure 2: $F-H$ diagram for the investigated soil (see also Fig1)

the skeleton. He applies Terzaghi's filter criterion and defined a self-filtration index $(I S F)$ as follow:

$I S F=d_{15, F} / d_{85, B}$

where $d_{15, F}$ is the skeleton particle diameter for which $15 \%$ of the grains by weight of the coarse particles are smaller, and $d_{85, B}$ stands for the embedded particle diameter for which $85 \%$ of the fine particles by weight are smaller.

By Witt's method, the procedure of separation is not necessary. The self-filtration index $(I S F)$ can be expressed by the slope of the original PSD. For the stability assessment following conditions must be considered:

1. Internal stability : $I_{S F} \leq 6$ or slope of PSD greater equal than 0.193

2. Internal instability : $I_{S F} \geq 9$ or slope of PSD less equal than 0.157

If the slope of the PSD is flatter than $15.7 \%$ for intervals of $H=15 \%$, the skeleton is not able to retain the embedded fine particles. This critical slope can be easily drawn as the increase of mass equal to $15.7 \%$ per decade in a half logarithmic standard gradation diagram. It must be mentioned that this criterion can be used for widely graded soils with a fine content less than $30 \%$. By Witt's criterion, there are two limit conditions: $I_{S F} \leq 6$ implies internal stability and $I_{S F} \geq 9$ implies definite internal instability. Between these two limits, the soil is in the transition zone and can obviously be either stable or unstable. A calculation example of the self-filtration index according to Witt (2013) computed for the investigated soil is shown in Figure3.

For the application of Witt's criterion, the soil has to possess a dominant coarse matrix (see Jentsch et al. 2014). The PSD of such a soil has to be split up at 


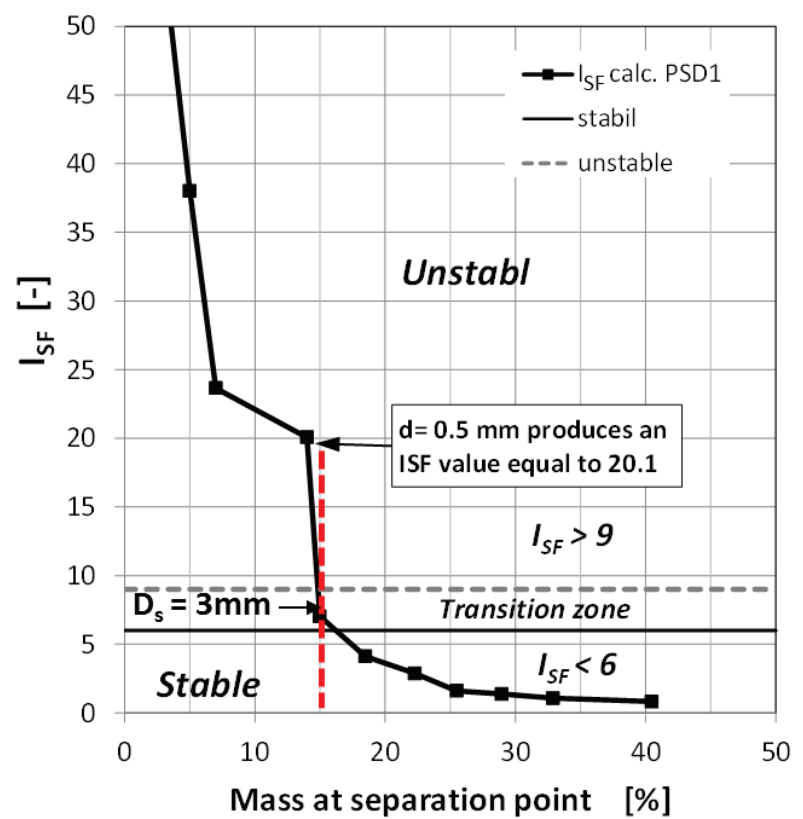

Figure 3: The course of the self filtration index according to Witt (2013) for the investigated PSD

different points and the resulting PSDs, i.e. filter and base, have to be checked against each other with respect to the filter rule of Terzaghi. Usually, the most unfavorable combination of base and filter is obtained if the split-off point or the separation point according to Salehi Sadaghiani and Witt (2012) lies in the range of a small inclination within a certain length of the PSD. Therefore, the split can be done, for example, at the $(H / F)_{\min }$. This can also be found by using the self-filtration index. The self-filtration index can be calculated for the intervals of $15 \%$ of mass for the selected PSD.

\subsection{Hydraulic suffusion criterion}

Istomina (1957) cited by Busch et al. (1993) carried out tests on various kinds of soil under vertical upward flow and proposed the estimation of the critical hydraulic gradient based on the coefficient of uniformity of the PSD. Skempton and Brogan (1994) also accomplished suffusion tests with upward flow and determined the critical hydraulic gradient in depen-

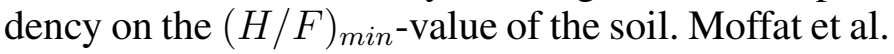
(2011) investigated the effect of the vertical load acting on the samples surface for unstable soils. They found that the critical gradients $\left(i_{c r i t}\right)$ increased with an increasing surface load.

By preliminary tests, the authors found out that upward flow tests for the soils with a fine content of more than $10 \%$ lead to uplift (hydraulic heave) of the sample because of a slight non-homogeneity of the sample. During sample placement of the widely graded soil, segregation of the sample has observed even using under-water-placement-technique. The segregation causes an accumulation of the finer particles at the bottom and between the sample layers during the placement. Thus, the hydraulic conductivity of the thin segregation layers is much lower than that of the sample layers between them. Therefore, the main decrease of the hydraulic head with a high local hydraulic gradient takes place within these thin segregation layers. Hence, an upward flow can lead to uplift and/or liquefaction on the top of the sample, which can result in an over- or underestimation of the critical hydraulic gradient.

In the case of liquefaction on the top of the sample, because of the uplift forces, the smaller particles are washed out. According to the definition, however, suffusion is a process which occurs in the soil mass so that there is no evidence of the washout on the top of the soil. Moreover, according to Moffat et al. (2011), a load on the top of the sample can increase the critical hydraulic gradient. Thus, the load on the sample surface can resist the uplift forces on the mentioned local stratification. The experimental setup using upward flow for finding the critical hydraulic gradient is not an easy task due to stated problems. It can lead to an underestimation of $i_{c r i t}$ for widely graded soils with more than $10 \%$ fines and to an overestimation of $i_{\text {crit }}$ for the soils with less than $10 \%$ of fines.

Due to the effect of the load acting on the sample surface, avoiding liquefaction and hydraulic heave for the investigated soil with a mass of circa $15 \%$ of fines, an experimental setup using downward flow was chosen.

\section{EXPERIMENTS}

\subsection{Test apparatus}

The test apparatus with a dimeter of $300 \mathrm{~mm}$ and an height of $800 \mathrm{~mm}$ was developed especially to study the widely gap graded soil (Fig.12). The large-scale device allows testing of various soils with a maximum particle size of $30 \mathrm{~mm}$ and different sample heights under optimized conditions. The PSD which is used for the suffusion tests is shown in Figure 1. The experimental setup used in this investigation is shown in Figure 4. More details about the experimental setup and test apparatus can be found in Salehi Sadaghiani and Witt (2011).

The two parts of the test cylinder allow the installation of different sample heights. A vertical stress up to $30 \mathrm{kN} \mathrm{m}^{-2}$ can be applied using the pneumatic pressure. The applied load prevents uplift during the saturation and the volume changes of the sample during the particle washout can be calculated. For the acquisition of potential changes within the soil structure 8 pressure sensors were attached. For additional visual control, an observation with piezometer pipes can give insight into clogging processes. A distance between the single pressure sensors of $5 \mathrm{~cm}$ permits a relatively detailed recording of the pore-water pressure changes along the soil column. The measurements along the soil column were carried out approximately at points with a distance of $10 \mathrm{~cm}$ from the 


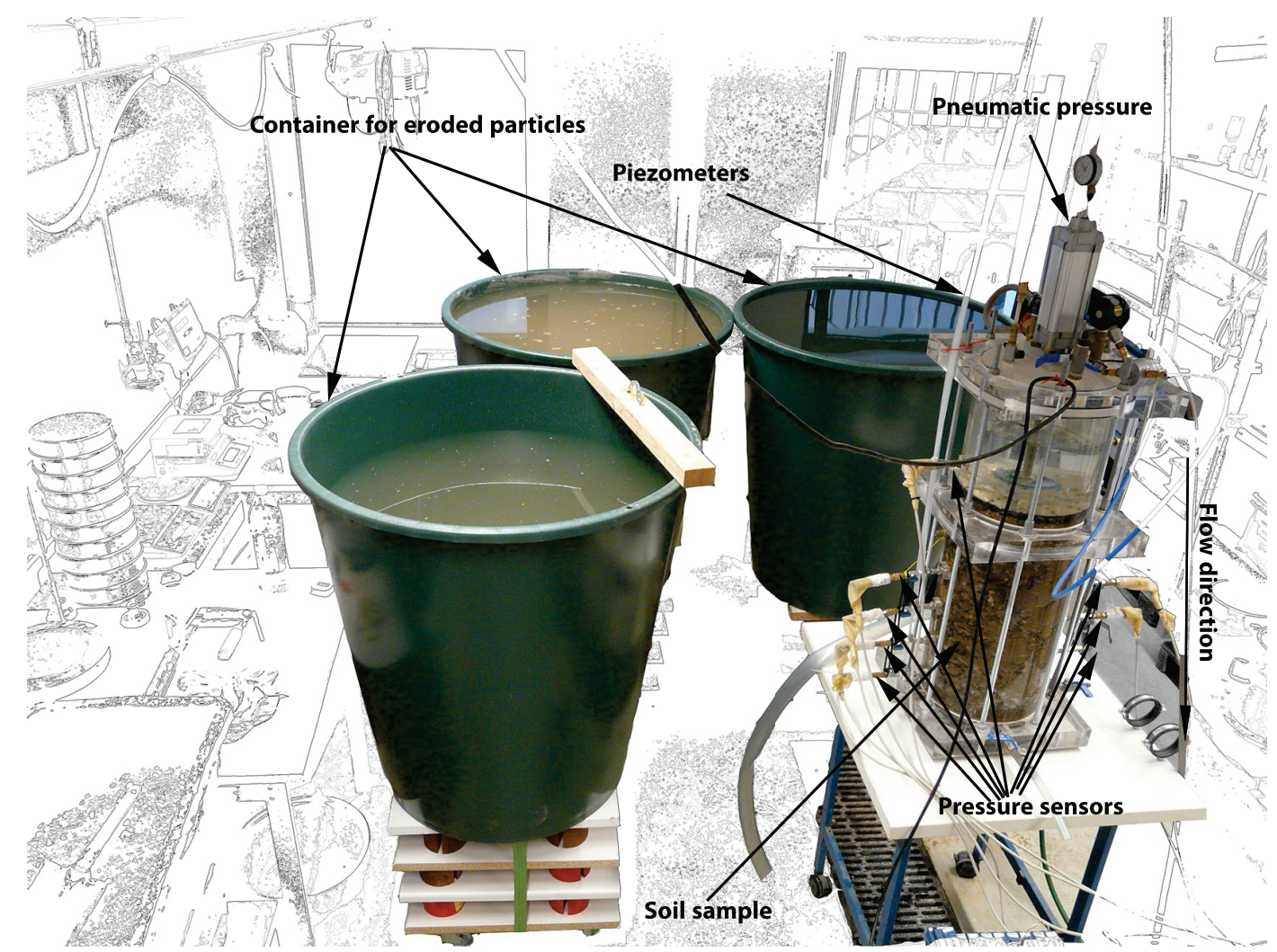

Figure 4: Experimental setup for qualitatively homogeneous suffusion tests - the total soil sample was sieved to its fractions and divided into 4 batches and placed carefully to achieve probabaly the most possible homogeneous sample.

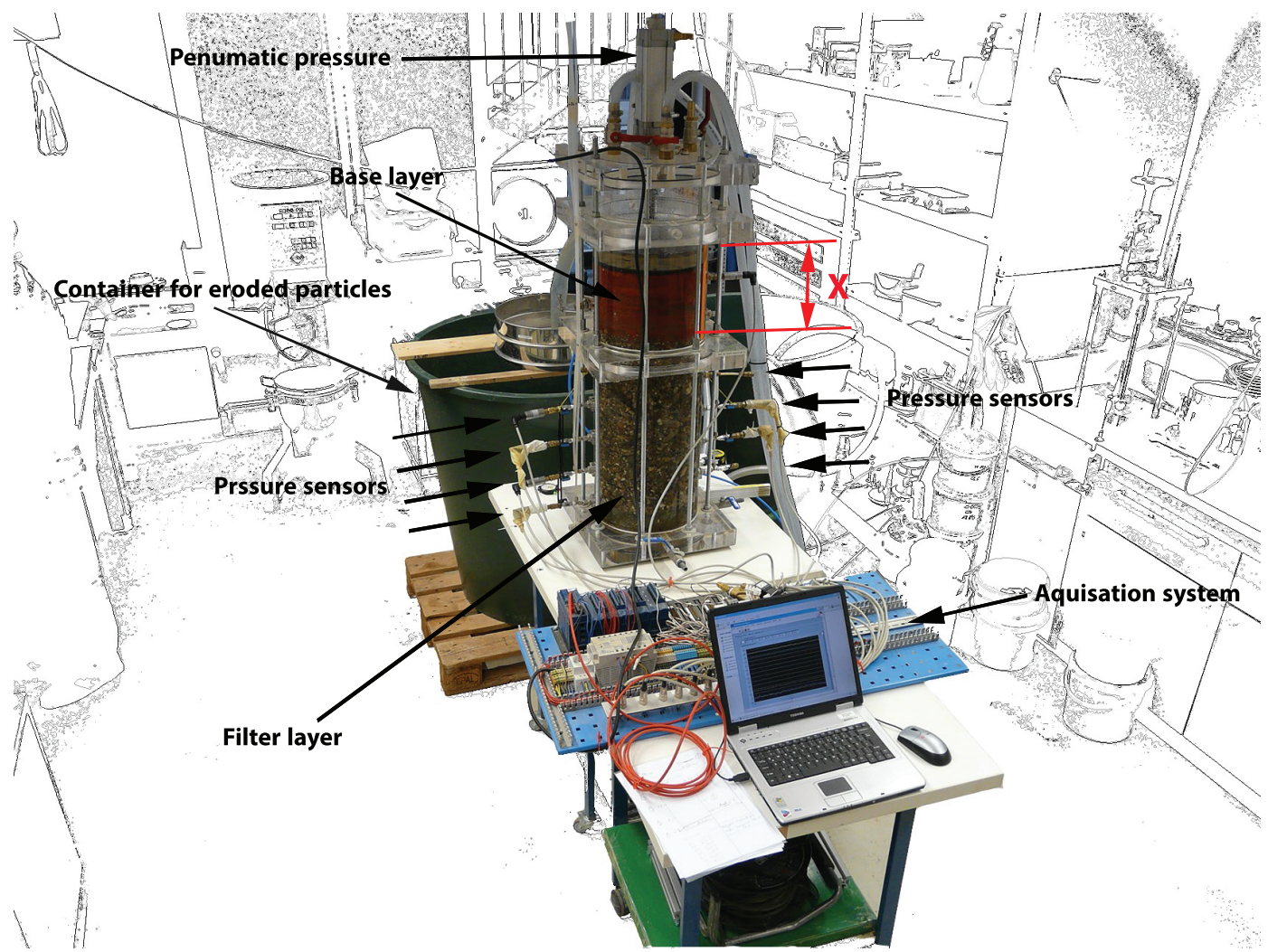

Figure 5: Experimental Setup for segregated suffusion tests - $\mathrm{X}$ is the height of the fines smaller than 2 mm above the soil skeleton $D_{\text {Skeleton }} \geq 2 \mathrm{~mm}$ with a separation point equal to $2 \mathrm{~mm}\left(D_{s}=2 \mathrm{~mm}\right)$. 4 segregated suffusion tests have been carried out with different heights $X=15,10,5$ and $2 \mathrm{~cm}$. 
center of the soil column, in order to exclude boundary effects.

\subsection{Suffusion tests (ST)}

By these tests local and global transport of mobile particles, applying high hydraulic gradients was measured. In each test, steady flow conditions inside the embankment were simulated. Structural changes within the sample were investigated by measurement of the pore-water pressure along the sample (see Fig.4 and Fig.5). The eroded particles were collected for the determination of the maximum size of suffusive particles. The measurement of the eroded mass at the outlet is a primary criterion for the identification of the sample stability. After each suffusion test $(S T)$, changes of the PSD inside of four various layers of the soil column were measured. This allows a conclusion about global and local particle movement. Suffusion test have been carried out using different sample preparation methods, which can be obtained form Table

The first six Suffusion Tests $(S T)$ have been conducted on samples built in by one layer using a shovel. A water content of $8 \%$ was applied to avoid substantial segregation.

The second series of suffusion tests $\left(S T_{h} \mathrm{om}\right.$.) with a sample height of about $500 \mathrm{~mm}$ were carried out using a reconstitution technique (see also Salehi Sadaghiani et al. 2012 and Fig.4). These samples were built in by layers with a thickness of about $125 \mathrm{~mm}$ (four layers) and were compacted. Each layer has been built in the test apparatus with the same specified PSD and the same weight of its fractions. This was to replicate the PSD in each soil layer and to find out the percentage of the transported fractions from layer to layer after the test. A reference layer of glass spheres with a diameter of 8 to $16 \mathrm{~mm}$ was built at the base of the sample for avoiding dynamical washout of particles during sample placement and compaction. Mesh grids with an opening size of $6 \mathrm{~mm}$ have been used below and above of the reference layer.

The third series of suffusion tests $\left(S T_{\text {seg. }}\right.$ ) have been conducted on the same samples according to the given PSD. However, the samples have been separated by a sieve of $2 \mathrm{~mm}$ and the finer part has been placed on the top of the coarser part (soil skeleton). These tests can be seen as a classical filtration test or contact erosion test.

The idea behind these tests was to create extremely segregated situation during placement so that the particles smaller than $2 \mathrm{~mm}$ sit above coarser part of the soil. All of the fine particles have been replaced with glass beads. For these tests $S T_{\text {seg. }}$, a sample consisted of glass-sand-gravel mixture was used. The weight of the sample for the test $\left(S T_{\text {seg. }} 1-\right.$ fine layer $\left.15 \mathrm{~cm}\right)$ is $78 \mathrm{~kg}$. More than $60 \%$ of the sample weight was the weight of the soil skeleton (ca. $60 \mathrm{~kg}$ ) and the weight of the fines on the top of the skeleton was $18 \mathrm{~kg}$ (see
Fig.6). This amount of fines built a $15 \mathrm{~cm}$ fine layer on the top of the soil skeleton according to the original PSD (see Fig. 5). Finally, a vertical load has been applied on top of the soil column. The sample has been saturated from bottom to top. The flow during the test was in the direction of the gravity. Different hydraulic gradients from $i=0.1$ to 53 have been applied to the same sample.

By test $\left(S T_{\text {seg. }} 1\right.$ - fine layer $\left.15 \mathrm{~cm}\right)$, no particle movement and no changes in the hydraulic conductivity could be observed. After this, the height of the fine layer has been reduced to $10 \mathrm{~cm}$, $5 \mathrm{~cm}$ and finally, $2 \mathrm{~cm}$. For those tests $\left(S T_{\text {seg. }} 2-\right.$ fine layer $10 \mathrm{~cm}),\left(S T_{\text {seg. }} 3\right.$ - fine layer $\left.5 \mathrm{~cm}\right)$ and $\left(S T_{\text {seg. }} 4\right.$ - fine layer $\left.2 \mathrm{~cm}\right)$, water has been percolated for a duration of five hours. The fine layer stayed unchanged for the thicknesses of $10 \mathrm{~cm}$ and $5 \mathrm{~cm}$ even with a very high hydraulic gradient of $i=0.53$. The fine layer has been eroded by the fine lyaer of $2 \mathrm{~cm}$ $\left(S T_{\text {seg. }}\right.$ 4). The layer with the thickness of $2 \mathrm{~cm}$ has a mass of $2.4 \mathrm{~kg}$. If we mix this amount of fine with the skeleton, we get a totally new PSD with a fine content of almost $5 \%$.

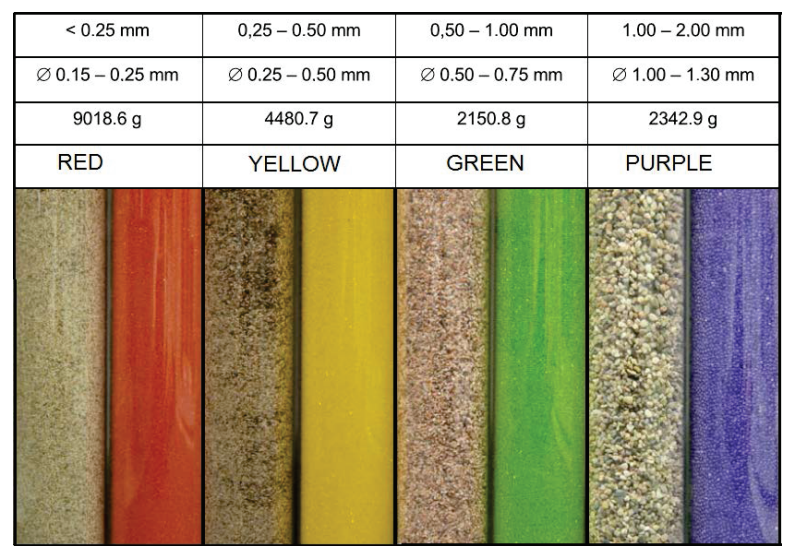

Figure 6: Type and amount of glass spheres used for the reconstitution of the original PSD. They also serve to identify the depth of particle transport into the soil skeleton.

In all the performed tests, the following parameters were measured (qualitatively or quantitatively):

1. visual inspection of the form of filtration (particle clogging, washing through),

2. measurement of the pore water pressure changes during the test in dependency on the hydraulic gradient,

3. measurement of mass and volume changes of the sample during the suffusion tests,

4. determination of mass of soil fractions in each layer after and before the tests,

5. measurements related to particle washout (mass, size and PSD of the washout), and

6. determination of local and global hydraulic gradients as well as filter velocity. 
Table 1: Suffusion tests with various types of sample placement

\begin{tabular}{lll}
\hline Suffusion Test & Number & Comment \\
\hline$S T$ & 6 & downward flow, sample placement in one layer with shovel \\
$S T_{\text {hom }}$ & 3 & downward flow, sample placement in 4 layers with exact the same PSD \\
$S T_{\text {seg. }}$ & 4 & downward flow, fine particles smaller than $2 \mathrm{~mm}$ on the top of the soil skeleton with \\
& & different thicknesses from $2 \mathrm{~mm}$ to $2 \mathrm{~mm}$ \\
\hline
\end{tabular}

\subsection{Critical hydraulic gradient $i_{\text {crit }}$}

For the determination of the critical hydraulic gradient $i_{\text {crit }}$ the water discharge has been measured and the filter velocity of the water flow was determined. For an internally unstable soil, the initiation of the particle washout depends on several factors. According to Patrašev (1957) cited by Lubockov (1965), the soil permeability and stability do not change significantly if the soil loses less than $3 \%$ of its mass. Thus, if the particle washout is higher than $3 \%$ of the total sample mass, and the filter velocity is continuously increasing without changing the hydraulic gradient, the sample can be evaluated as internally unstable. If the particle washout is less than $3 \%$ of the entire sample mass and the discharge does not change drastically for a constant hydraulic gradient, the sample can be evaluated as internally stable.

\section{RESULTS}

The filter velocity and the mass of particle washout in relation to hydraulic gradient for $S T$ s (sample with one layer of $500 \mathrm{~mm}$ ) are given in Fig.7 and Fig.8.

As a matter of fact, widely graded soils tend to segregate and trying to place soil, particle by particle, in a test apparatus does not replicate the in-situ conditions. Therefore, the objective of the experiments was to represent the significant effect of heterogeneity even in laboratory scale.

The percentile mass of particle washout is given by the following formula.

$m=m_{i} / m_{\text {total }}$

$m_{r}=m_{i} /\left(m_{i, \min }\right)$

where $m$ is the mass of particle washout, $m_{i}$ indicates the mass of particle washout at the end of each test step with a constant hydraulic gradient, $m_{i, \min }$ stands for the minimum mass amount of particle washout, and $m_{\text {total }}$ the total mass of the sample before starting the test.

Table 2 and Table 3 give some detailed results of the STs. Table 2 shows an example of the percentile mass of particle washout for a hydraulic gradient of $i=0.1$ for single layer samples (for the definition of the parameters see also equation 2 and 3). In different $S T \mathrm{~s}$, various amounts of mass were washed out. The maximum washout mass exceeds the minimum washout mass by $440 \%$. This implies a very high standard deviation. Here, the calculated standard deviation is not suitable to describe the distribution of the measured results, because they do not

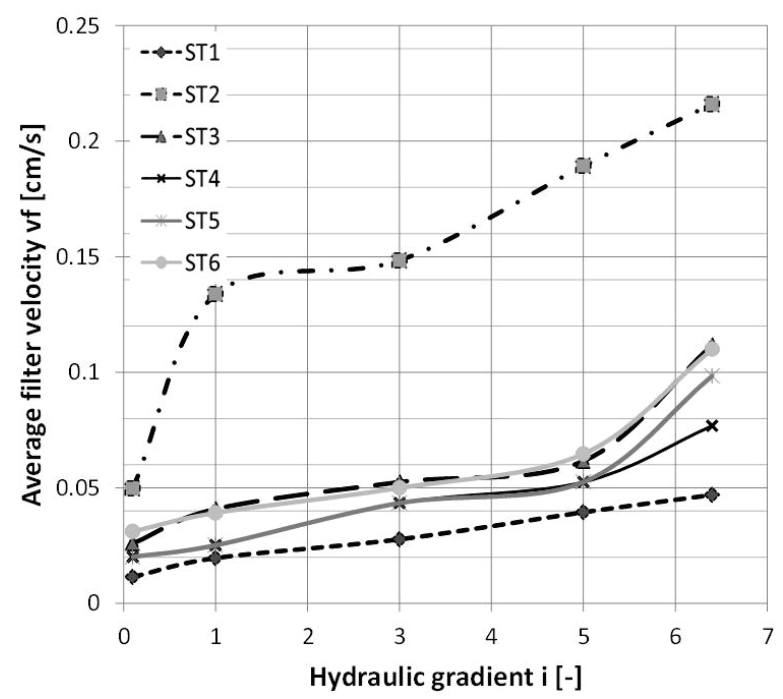

Figure 7: Development of filter velocity with increasing hydraulic gradient for single layer test samples

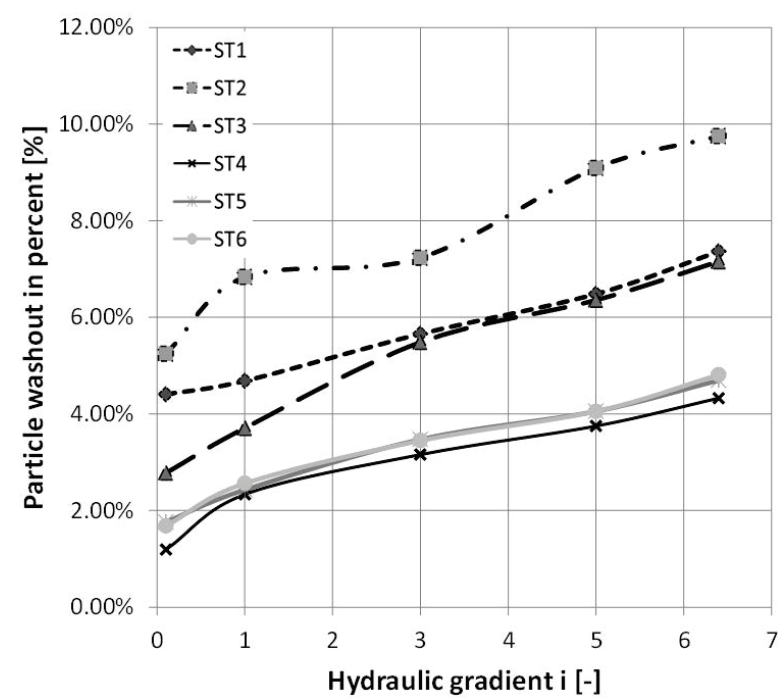

Figure 8: Development of the mass of particle washout with increasing hydraulic gradient for single layer test samples

sufficiently match to a normal or log-normal distribution using various statistical normality tests such as KolmogorovSmirnov test or quantile-quantile plot (Cleveland et al. 1985 and James 2006). This kind of result with an apparently high standard deviation is often reported in the literature (Kenney and Lau 1985; Wan and Fell 2008 and Ahlinhan et al. 2011). One method of analyzing this kind of data is to eliminate outliers out of the basic statistic population.

The results of the second series of suffusion tests (SThom.) are presented in Table 4, Figure 9 and Figure 10.

The following Figures 11,12, 13 and 14 illustrate some details of particle mobilization and transportation in the segregated suffusion tests $\left(S T_{\text {seg. }}\right)$. In the $\left(S T_{\text {seg. }} 4\right)$, the fine layer has a thickness of $2 \mathrm{~cm}($ 
Table 2: Two extreme cases of material transport for $S T$ s with single layer placement technique.

\begin{tabular}{|c|c|c|c|c|}
\hline \multirow[b]{2}{*}{$\mathrm{i}[-]$} & \multicolumn{2}{|l|}{$S T 2$} & \multicolumn{2}{|l|}{ ST4 } \\
\hline & $m[\%]$ & $v_{f}[\mathrm{~cm} / \mathrm{s}]$ & $m[\%]$ & $v_{f}[\mathrm{~cm} / \mathrm{s}]$ \\
\hline 0.1 & 5.25 & 0.0497 & 1.19 & 0.02014 \\
\hline 1.0 & 6.84 & 0.1337 & 2.33 & 0.02513 \\
\hline 3.0 & 7.23 & 0.1484 & 3.16 & 0.04340 \\
\hline 5.0 & 9.09 & 0.1892 & 3.75 & 0.05249 \\
\hline 6.4 & 9.75 & 0.2160 & 4.33 & 0.07681 \\
\hline
\end{tabular}

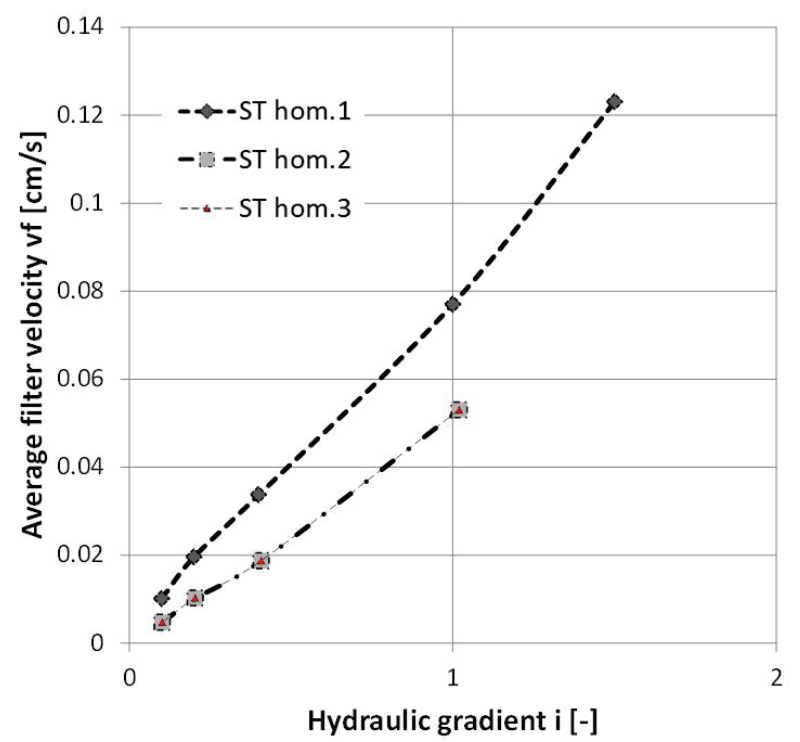

Figure 9: Development of filter velocity with increasing hydraulic gradient for test samples build in by four layers using reconstitution technique.

Fig.14). This thickness of the fine layer was not sufficient to resist a hydraulic gradient of $i=5.5$. The seepage flow caused a hole in this layer, and particle mobilization has been initiated. It took a long time till the mobilized particles were washed out totally through the sample (circa 25 minutes). After a washout of $0.08 \%$ (51.8 g) of the total washout mass, a constant discharge was reached, i.e. the measured hydraulic conductivity for the rest of the test duration of 60 minutes was constant. The hydraulic gradient was increased up to $i=11.0$. Remobilization of particles has been observed, but no particle washout could be measured. The step-wise increase of the hydraulic gradient has been done up to the maximum possible hydraulic gradient of $i=53.0$. After 60 minutes of water percolation only $1.21 \mathrm{~g}$ of fines were washed out. In Figure 13, the surface of each layer after the

Table 3: Comparison of particle washout by applying a hydraulic gradient of $i=0.1$ for the test with single layer placement technique

\begin{tabular}{lll}
\hline Test Nr. & $m[\%]$ & $m_{r}[\%]$ \\
\hline$S T 1$ & 4.405 & 370 \\
$S T 2$ & 5.246 & 440 \\
$S T 3$ & 2.778 & 233 \\
$S T 4$ & 1.190 & 100 \\
$S T 5$ & 1.763 & 148 \\
$S T 6$ & 1.684 & 141 \\
\hline $\max$ & 5.246 & 440 \\
$\min$ & 1.190 & 100 \\
\hline
\end{tabular}

Table 4: Test results for $S T_{h o m} \mathrm{~s}$.

\begin{tabular}{|c|c|c|c|c|}
\hline \multirow[b]{2}{*}{ Test Nr. [-] } & \multicolumn{2}{|c|}{$i=0.1$} & \multicolumn{2}{|c|}{$i=1.0$} \\
\hline & $\mathrm{m}[\%]$ & $m_{r}[\%]$ & $\mathrm{m}[\%]$ & $m_{r}[\%]$ \\
\hline SThom.1 & 0.41 & 154 & 6.06 & 207 \\
\hline SThom.2 & 0.27 & 100 & 4.64 & 159 \\
\hline SThom.3 & 0.73 & 275 & 2.92 & 100 \\
\hline $\max$ & 0.73 & 275 & 6.06 & 207 \\
\hline $\min$ & 0.27 & 100 & 2.92 & 100 \\
\hline
\end{tabular}

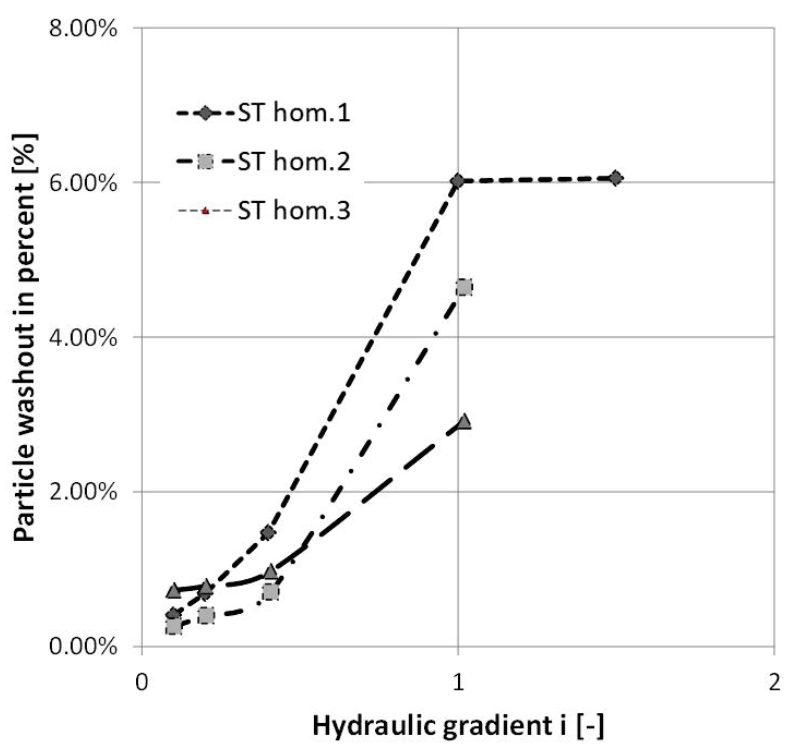

Figure 10: Development of the mass of particle washout with increasing hydraulic gradient for single layer test samples

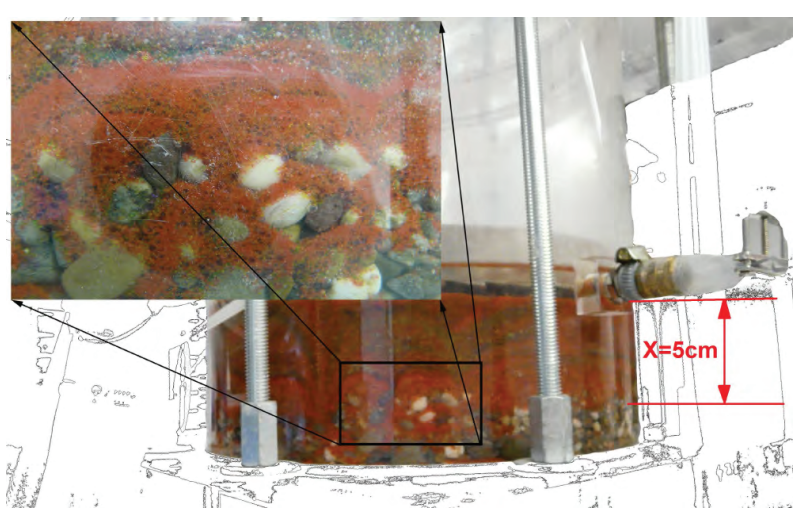

Figure 11: Detailed view of the segregated suffusion tests $\left(S T_{\text {seg. }}\right)$ with the fine layer of $2 \mathrm{~cm}$. This amount of fine was not sufficient to resist a hydraulic gradient of $i=5.5$. The seepage flow caused a hole in this layer and particle transport started.

suffusion test and the depth of the particle penetration are shown. The fine particles have been transported through the sample (washed out). In Figure 14, it can be seen that the fine particles of the segregated sample can only be mobilized with a very high hydraulic gradient of $i=5.5$. This amount of fine represents the original skeleton of the soil plus $4 \%$ of fines.

The size of the suffusive particles has been measured from the discharge. The biggest suffusive particles belonged to the soil fractions with a grain diameter between 0.125 and $0.25 \mathrm{~mm}$. Additionally, the mobile particles were determined by balancing the weight of the different fractions of each soil layer. The largest mobile particles within the soil column had a diameter between 0,5 and $2 \mathrm{~mm}$. It can be stated that in practical terms, it is too conservative to assume that 


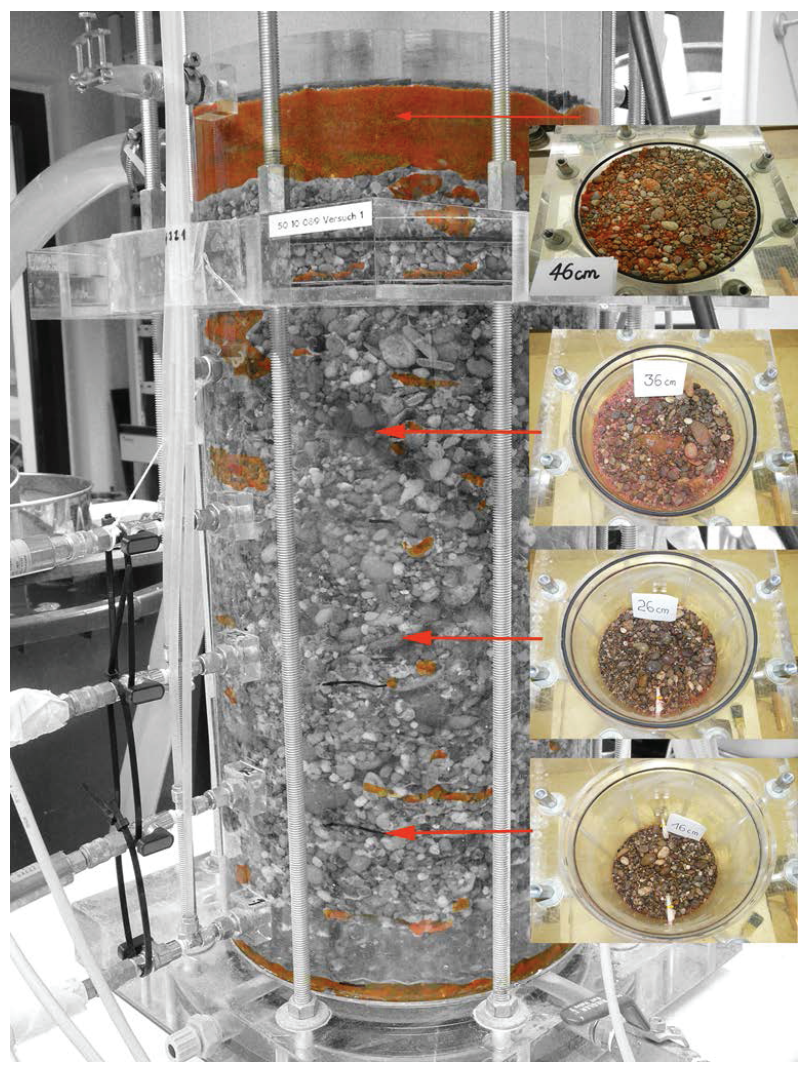

Figure 12: Detailed view of the segregated suffusion tests $\left(S T_{\text {seg. }}\right.$. $)$ with the fine layer of $2 \mathrm{~cm}$. After the tests the sample was sieved layer by layer to find the mass of fines transported into the pores of the skeleton before reducing the hydraulic conductivity.

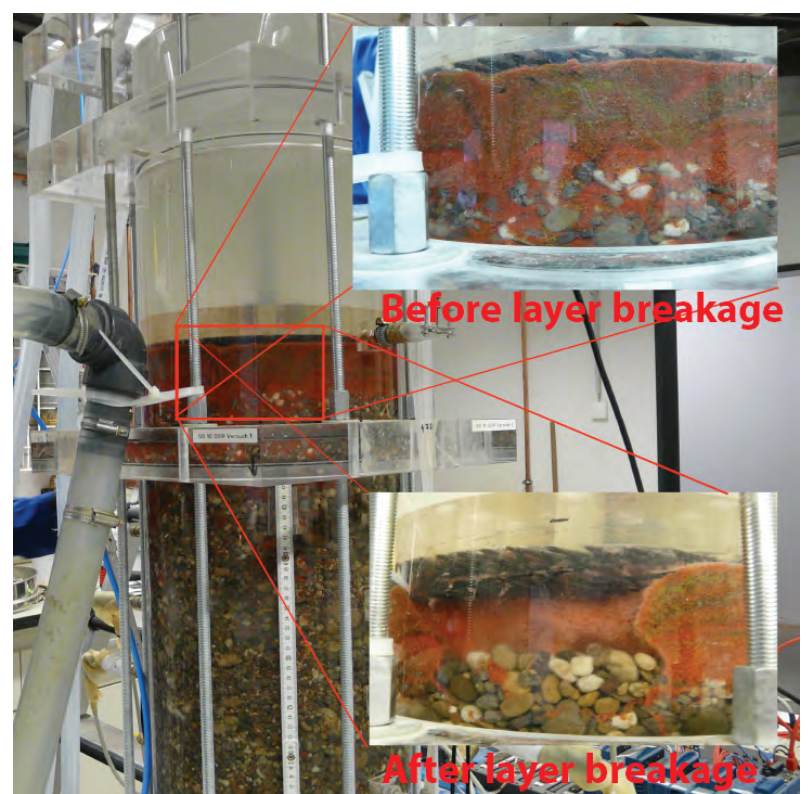

Figure 13: Detailed view of the segregated suffusion tests $\left(S T_{\text {seg. }}\right.$. $)$ with the fine layer of $2 \mathrm{~cm}$. This amount of fine was not sufficient to resist the hydraulic gradient of $i=5.5$. The seepage flow caused a hole in this layer and particle transport started.

the whole finer fractions of an unstable widely graded soil can be eroded. The results of the $S T_{\text {seg. }}$ show that a big number of particles smaller than $2 \mathrm{~mm}$ are mobile but if the sample is large enough, they cannot go through the pore channels, and the seepage forces are not sufficient to re-mobilize them, even for increased hydraulic gradients. The results of the $S T_{\text {seg. }}$ s show that more than $99 \%$ of the fine particles of the PSD

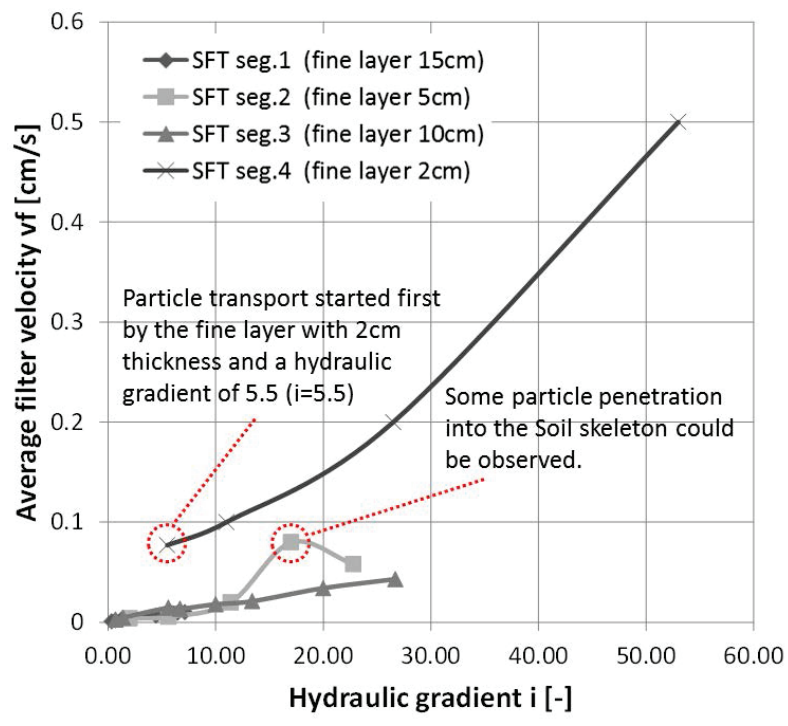

Figure 14: Development of the filter velocity with increasing hydraulic gradient for segregated suffusion tests

are mobile, but these particles are not suffusive particles. The findings of the $S T_{\text {seg. }}$ s indicate that less than $1 \%$ of the fine particles smaller than $2 \mathrm{~mm}$ are suffusive.

\section{CONCLUSION}

The results of the reported experiments show the insufficiency of an overall hydraulic gradient as a criterion for the suffusion risk of widely graded soils. Because of the inherent variability of the particle arrangement in widely graded soils, a global hydraulic gradient cannot be a significant factor for the initiation of suffusion. The local gradients which were measured using pressure sensors show that there are variations of the local hydraulic gradient by several orders of magnitude according to the particle arrangement in different samples.

It has been demonstrated that a rough estimation of $i_{\text {crit }}$ can be determined based on variations of seepage velocity, hydraulic gradient, and soil homogeneity. It could be observed that the required value of the $i_{\text {crit }}$ for the initiation of suffusion changes with the variation of the sample placement technique. This implies a considerable impact of the homogeneity, and the fact that homogeneity cannot be assumed even in laboratory scale. Thus, it is necessary to study the suffusion process within a certain size of a Representative Elementary Volume (REV) under a controlled effective stress subject to its local and global variability.

It is also shown that even for a sample with a high degree of homogeneity the obtained results can vary sharply. The observations in the different test series indicate that there are two group of particles which do not transmit the stresses. The first group is the mobile particles in the samples which are not fixed and the second group is the suffusive particles, which can be transported and washed out of the sample. They can have the same size, but the pore constrictions along the pore channels of the soil skeleton decide if a par- 
ticle is suffusive or mobile. The seepage forces cause a change of the original structure into a sometimes more or sometimes less stable one.

It can be stated that the most challenging problem in assessing the erosive processes is to determine the degree of homogeneity for a widely graded soil. If the level of the homogeneity cannot be determined with an appropriate accuracy laboratory tests as described above can give some insight about the maximum and minimum hydraulic gradients. Minor differences in the shape of the PSD affect the internal stability of the soil, and it is recommended for significant earthworks to carry out laboratory tests to confirm the assessments made upon geometrical and hydraulic criteria.

\section{ACKNOWLEDGMENT}

This research is a part of a project that funded by German waterways and shipping office Freiburg (WSA). The authors wish to thank the WSA-Freiburg for the financial and technical supports.

\section{REFERENCES}

Ahlinhan, M., M. Achmus, S. E. Burns, S. K. Bhatia, C. M. Avila, B. E. Hunt, et al. (2011). Experimental investigation of critical hydraulic gradients for unstable soils. In Scour and erosion. Proceedings of the Fifth International Conference on Scour and Erosion (ICSE5), San Francisco, California, USA, 7-10 November, 2010., pp. 599-608. American Society of Civil Engineers (ASCE).

Bonelli, S. (2012). Erosion of geomaterials. John Wiley \& Sons.

Burenkova, V. V. (1993, 20-22 October). Assessment of suffusion in non-cohesive and graded soils. In U. J. Brauns, M. Heibaum (Ed.), Filters in Geotechnical and Hydraulic Engineering, First International Conference "Geo-Filters", Karlsruhe, Germany, pp. 357-360. A.A Balkema, Rotterdam, Brookfield.

Busch, K.-F., L. Luckner, \& K. Tiemer (1993). Geohydraulik. Gebrüder Borntraeger.

Chapuis, R. P. (1992). Similarity of internal stability criteria for granular soils. Canadian Geotechnical Journal 29(4), 711-713.

Cleveland, W. S. et al. (1985). The elements of graphing data. Wadsworth Advanced Books and Software Monterey, CA.

Istomina, V. (1957). Filtration stability of soils. Gostroizdat, Moscow, Leningrad 15.

James, F. (2006). Statistical methods in experimental physics. World Scientific.

Jentsch, H., M. R. S. Salehi Sadaghiani, P. Winkler, \& K. Witt (2014). Experimental investigation-influence of the shape of the gradation curve on the soil structure. In Scour and Erosion: Proceedings of the 7th International Conference on Scour and Erosion, Perth, Australia, 2-4 December 2014, pp. 105. CRC Press.

Kenney, T. C. \& D. Lau (1985). Internal stabilty of granular filters. Canadian Geotechnical Journal 22, 215.-225. article.

Kenney, T. C. \& D. Lau (1986). Internal stability of granular filters: Reply. Canadian Geotechnical Journal 23(3), 420-423.

Kenney, T. C. \& J. Westland (1993). Labratory study of segregation of granular filter materials. Filters in Geotechnical and Hydraulic Engineering, 313-319.

Kézdi, A. (1979). Soil physics-selected topicsdevelopments in geotechnical engineering-25. Technical report.

Lubockov, E. (1965). The calculation of suffosion properties of non-cohesive soils when using the non-suffosion analogue. In Proceedings of the International Conference on Hydraulic Research, Brno, Czech Republic, pp. 135-148.

Marot, D., F. Bendahmane, \& J.-M. Konrad (2011). Multichannel optical sensor to quantify particle stability under seepage flow. Canadian Geotechnical Journal 48(12), 1772-1787.

Moffat, R., R. J. Fannin, \& S. J. Garner (2011). Spatial and temporal progression of internal erosion in cohesionless soil. Canadian Geotechnical Journal 48(3), 399-412.

Patrašev, A. (1957). Motedika podbora granulometričeskogo sostava obratnych fil'trov. Sbornik trudov Lengiprorečtransa.

Perzlmaier, S. (2007). Verteilte Filtergeschwindigkeitsmessung in Staudämmen. Lehrstuhl für Wasserbau und Wasserwirtschaft im Inst. für Wasserwesen.

Reboul, N., E. Vincens, \& B. Cambou (2010). A computational procedure to assess the distribution of constriction sizes for an assembly of spheres. Computers and Geotechnics 37(1), 195-206.

Richards, K. S. \& K. R. Reddy (2007). Critical appraisal of piping phenomena in earth dams. Bulletin of Engineering Geology and the Environment 66(4), 381-402.

Salehi Sadaghiani, M., K. Witt, \& H. Jentsch (2012). A statistical approach to identify mobile particles of widely graded soils. In 6th International Conference of Scour and Erosion, ICSE, pp. 6-049.

Salehi Sadaghiani, M. R. \& K. J. Witt (2011). Experimental identification of mobile particles in suffusible non cohesive soils. European Journal of Environmental and Civil Engineering 15(8), 1155-1165.

Salehi Sadaghiani, M. R. \& K. J. Witt (2012, August 2731). Analysis of internal stability of widely graded soils based on identification of mobile particles. In 6th International Conference of Scour and Erosion, pp. 257264.

Saucke, U., J. Brauns, \& S. Wibel (1999). Zur Entmischungsneigung krniger Schttstoffe. Geotechnik 22(4), 259.-268.

Sherard, J. L., L. Dunnigan, \& R. S. Decker (1977). Some engineering problems with dispersive clays. In Dispersive Clays, Related Piping, and Erosion in Geotechnical Projects. ASTM International.

Sherard, J. L. \& L. P. Dunnigan (1989). Critical filters for impervious soils. Journal of Geotechnical Engineering 115(7), 927-947.

Skempton, A. \& J. Brogan (1994). Experiments on piping in sandy gravels. Geotechnique 44(3), 449-460.

To, H. D., A. Scheuermann, \& D. J. Williams (2012). A new simple model for the determination of the pore 
constriction size distribution. In 6th International Conference on Scour and Erosion (ICSE-6), pp. 295-303. Société Hydrotechnique de France (SHF).

Wan, C. F. \& R. Fell (2008). Assessing the potential of internal instability and suffusion in embankment dams and their foundations. Journal of Geotechnical and Geoenvironmental Engineering 134 No. 3, 401-407.

Witt, K. J. (2013). Der Selbstfiltrationsindex als Suffosionskriterium für nichtbindige Erdstoffe. Geotechnik 36(3), 160-168.

Ziems.J. (1969). Beitrag zur Kontakterosion nichtbindiger Erdstoffe. ,diss.,, TUDresden. 